\title{
An INTEGRAL View of the Spectral Energy Distribution of Major Flaring in Cygnus X-3
}

\author{
K. I. I. Koljonen* \\ CfA/SAO/Univ. of Helsinki \\ E-mail: karri.koljonenegmail.com \\ M. L. McCollough \\ CfA/SAO/CXC \\ E-mail: mmccollough@efa.harvard.edu
}

Cygnus X-3 is one of the brightest X-ray and radio sources in the Galaxy and was discovered in 1967 with the beginning of space-based X-ray astronomy. However after many years of observations in various wavelength bands Cygnus X-3 still eludes clear physical understanding.

To probe the physical properties of the system during its brightest radio state (a major radio flare), an extensive international, multiwavelength campaign was launched including $\mathrm{ToO}$ INTEGRAL observations with supporting observations in soft X-rays, UV, infrared and radio. We show that the spectral energy distributions (SEDs) constructed from these observations can be modeled with reddened synchrotron radiation together with an absorbed multi-color disk black body, Comptonization and reflection component. Different Comptonization models were studied and are discussed. In addition, we examine how the SEDs evolve with the major radio flares.

7th INTEGRAL Workshop

September, 8-11 2008

Copenhagen, Denmark

\footnotetext{
* Speaker.
} 


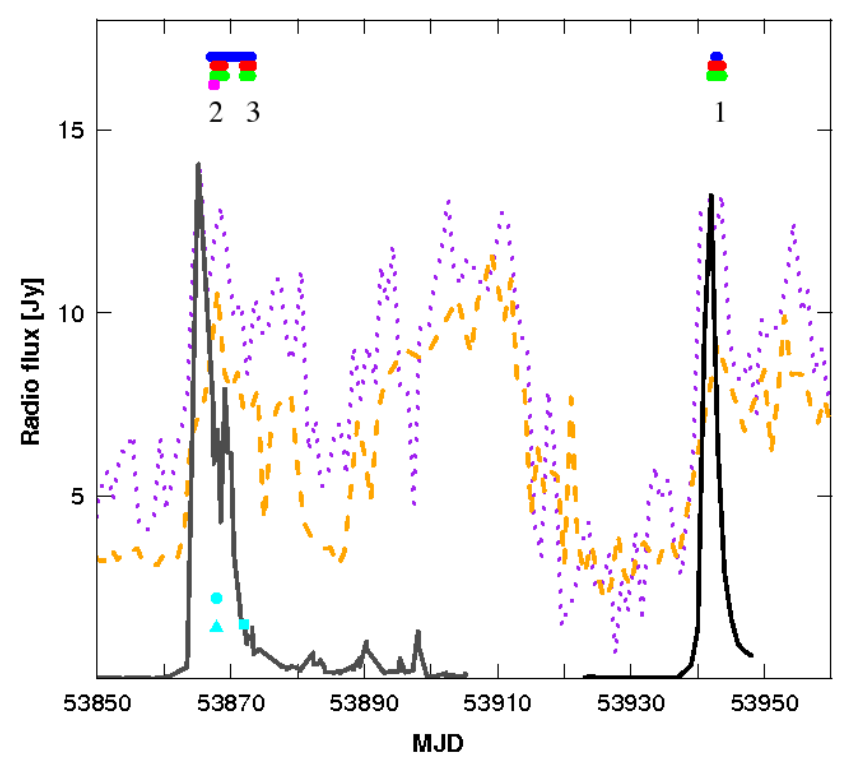

Figure 1: The data of the campaign. The grey solid line is the Ryle $15 \mathrm{GHz}$, the black solid line is the RATAN-600 2.3 GHz flux. The filled cyan circle, triangle and square are detections of the Nobeyama Millimeter Array at 98 and $110 \mathrm{GHz}$ and Yamaguchi Radio Telescope at $8.4 \mathrm{GHz}$ respectively (Tsuboi et al. 2008). Overplotted are the RXTE/ASM hardness (5-12 keV/3-5 keV, purple short-dash line) and Swift/BAT lightcurve (15-50 keV, orange long-dash line). The epochs of the X-ray and infrared observations are marked in the upper part of the figure (blue for RXTE, red for INTEGRAL, green for Swift and pink for PAIRITEL). The INTEGRAL observations labeled Obs. 1 (MJD 53943), Obs. 2 (MJD 53868) and Obs. 3 (MJD 53872), are numbered according to the time elapsed since the peak of a major flare $(0.25,2.51$ and 6.88 days respectively).

\section{Introduction}

Cygnus X-3 is a well known X-ray binary, its discovery dating back to 1967 (Giacconi et al. 1967). However, the nature of the system has remained a mystery despite extensive X-ray and radio observations throughout the years. Cygnus X-3 is thought to consist of a compact object orbiting a very massive and active helium star (a Wolf-Rayet star, van Keerkwijk et al. 1992) located at a distance of $\sim 9 \mathrm{kpc}$ in the plane of the Galaxy placing it in the Outer Arm (Predehl et al. 2000). In addition, Cygnus X-3 exhibits major radio flares which have been associated with jets extending from the compact object (e.g. Martí et al. 2000).

\section{Observations}

In May-July 2006, an extensive international campaign was undertaken during and following two major radio flares (13.8 Jy in the $15 \mathrm{GHz}$ band for the first flare and 13.2 in the $2.3 \mathrm{GHz}$ band for the second flare). This included observations across the electromagnetic spectrum from three 
space-based (INTEGRAL, Swift and RXTE) and three ground-based observatories (Ryle, RATAN600 and PAIRITEL). The main blocks of observing time are centered shortly after the peak of each major flare, ranging from 0.25 days to 6.88 days after the flare. The observations can be seen in Fig. 1. The INTEGRAL observations labeled Obs. 1 (MJD 53943), Obs. 2 (MJD 53868) and Obs. 3 (MJD 53872), are numbered according to the time elapsed since the peak of a major flare $(0.25$, 2.51 and 6.88 days respectively). Due to an overlap in energy range with INTEGRAL, the RXTE observations were omitted here but are included in Michael McCollough's contribution to these proceedings and will be examined more closely in Koljonen et al. (in prep.).

\subsection{Radio, UV and IR}

We use Ryle $15 \mathrm{GHz}$ data, Nobeyama Millimeter Array 98 and $110 \mathrm{GHz}$ data and Yamaguchi Radio Telescope $8.4 \mathrm{GHz}$ data (Tsuboi et al. 2008) simultaneous with the X-ray data after the first major flare (i.e Obs. 2 and Obs. 3 in Fig. 1). For the X-ray data after the second major flare (i.e Obs. 1 in Fig. 1) we use simultaneous RATAN-600 data from bands 2.3, 4.8, 7.7, 11.2, 21.7 and $30 \mathrm{GHz}$. We use the upper limits obtained by UVOT (Roming et al., 2005) on-board Swift in U, V, $\mathrm{W} 1$ and $\mathrm{W} 2$ bands when available. For Obs. 2 we also have simultaneous PAIRITEL observations in $\mathrm{J}, \mathrm{H}$, and $\mathrm{K}$ bands.

\subsection{X-rays}

\subsubsection{INTEGRAL}

For INTEGRAL we use JEM-X (Lund et al. 2003), IBIS/ISGRI (Lebrun et al. 2003) and SPI (Vedrenne et al. 2003) data from revolutions 437, 438 and 462 with exposure time of $\sim 100 \mathrm{ks}$ per observation. The spectra were analyzed using the Offline Science Analysis (OSA) version 7.0 (Courvoisier et al. 2003). For the JEM-X data we used the energy range 4.0-20.0 keV. For IBIS we used the energy range 20.0-200.0 keV and added 5\% systematic error to all channels. For SPI the spectra were extracted using the SPIROS program (Skinner \& Connell 2003) using a background model based on the mean count modulation of the detector array. For the energy range we used 20.0-100.0 keV with 25 logarithmic bins and 100.0-2000.0 keV with 5 logarithmic bins and added a $5 \%$ systematic error to all channels.

\subsubsection{Swift}

For Swift we use XRT (Burrows et al. 2005) observations simultaneous with the INTEGRAL ones with exposure time of $\sim 7 \mathrm{ks}$ per observation. The data was extracted using HEASOFT version 6.2. We use the energy range 1.0-8.0 keV for XRT.

\section{Data analysis}

All the data fitting was performed using ISIS (Houck 2002), which is one of the best available spectral fitting program that is well designed to handle multiwavelength data sets. The strategy of the fitting is as follows: We start by fitting the X-ray data, since most of the data points lie in the X-ray band, with a specific model without the synchrotron component and its associated interstellar reddening. Subsequently, all the resultant X-ray fit parameters were frozen except for 
the normalizations and interstellar reddening and synchrotron components were included and the model was fitted again. Once a suitable fit was found, all the parameters were thawed and allowed to be refit and the error determinations to be made. The SEDs are shown in Fig. 2.

McCollough et al. (1999) noted a positive correlation between the hard X-ray and the radio emission in the flaring state which suggests that the same electron distribution could be responsible for the emission in both bands. However, when the whole data set was fit with only a synchrotron model (sync in the non-thermal module by Houck \& Allen 2006) the radio could not be made compatible with the hard X-rays. The radio slope didn't match the hard X-ray slope and they are offset so that a simple break in the spectrum would not explain the observations. Because of this difference, different emission processes are likely at work. Therefore, we chose to model the SEDs with synchrotron radiation from the electrons in the jet and inverse Comptonization of the soft photons from the accretion disk in the base of the jet (corona), providing the previously observed link between the radio and the hard X-rays. Additionally, we included wholly and partially absorbed emission from the accretion disk, the three most prominent emission lines (Fe, $\mathrm{S}$, $\mathrm{Si}$ ) and an absorption edge of the ionized iron in the fit and attenuated the model with interstellar reddening. We used four different Comptonization models in our fits to probe different parameter spaces, these being comptt (Titarchuk 1994), compps (Poutanen \& Svensson 1996), reflion (Ross \& Fabian 2005; Ross, Fabian \& Young 1999) and eqpair (Coppi 1992, 1999). reflion was only used for providing an approximation for the ionization parameter used in compps and eqpair. The disk emission is included in compps and eqpair but not in comptt. Thus, we added an additional component, ezdiskbb (Zimmerman et al. 2005), for the comptt model. In the case of eqpair, we modeled both the powerlaw acceleration and mono-energetic injection and we included an additional black body component for the non-scattered part of the seed photons as recommended in Coppi (1999). See Table 1 for selected parameters.

\section{Discussion and conclusions}

Table 1 shows that all the Comptonization models, together with an absorbed disk black body, appropriate line emission and an edge, give rather good reduced chi-squared values (ranging from 0.99 to 1.77 ). This indicates that Comptonization is likely the most probable physical process driving the observed emission. Including the synchrotron model gives more weight to the physical processes in the jet, as compared to just including a power-law in the fit. The only infrared observation in this data set (Obs. 2) gives us crucial information on how energetic the jet is during the major flare, i.e. it allows us to see if the non-thermal signature of the synchrotron emission extends to the infrared region. We can also see a rapid drop of the SED in this region (around $0.001 \mathrm{keV}$ in Fig. 2), which can be modeled with a rather high value of interstellar reddening, $E(B-V) \sim 5.2$. However, this is compatible with the high values of absorption that are seen in the soft X-rays. The best fits were obtained when synchrotron component was allowed to go all the way to the soft X-rays.

Cygnus X-3 is notorious for having a complicated absorption profile (see Hjalmarsdotter et al. 2008 and Szostek \& Zdziarski 2008). This absorption most probably consists of contributions from at least three components: interstellar absorption $\left(N_{H} \sim 1.5 \times 10^{22} \mathrm{~cm}^{-2}\right.$, Dickey \& Lockman 1990), a local cloud (McCollough et al., in prep.) and intrinsic absorption from the stellar wind of 


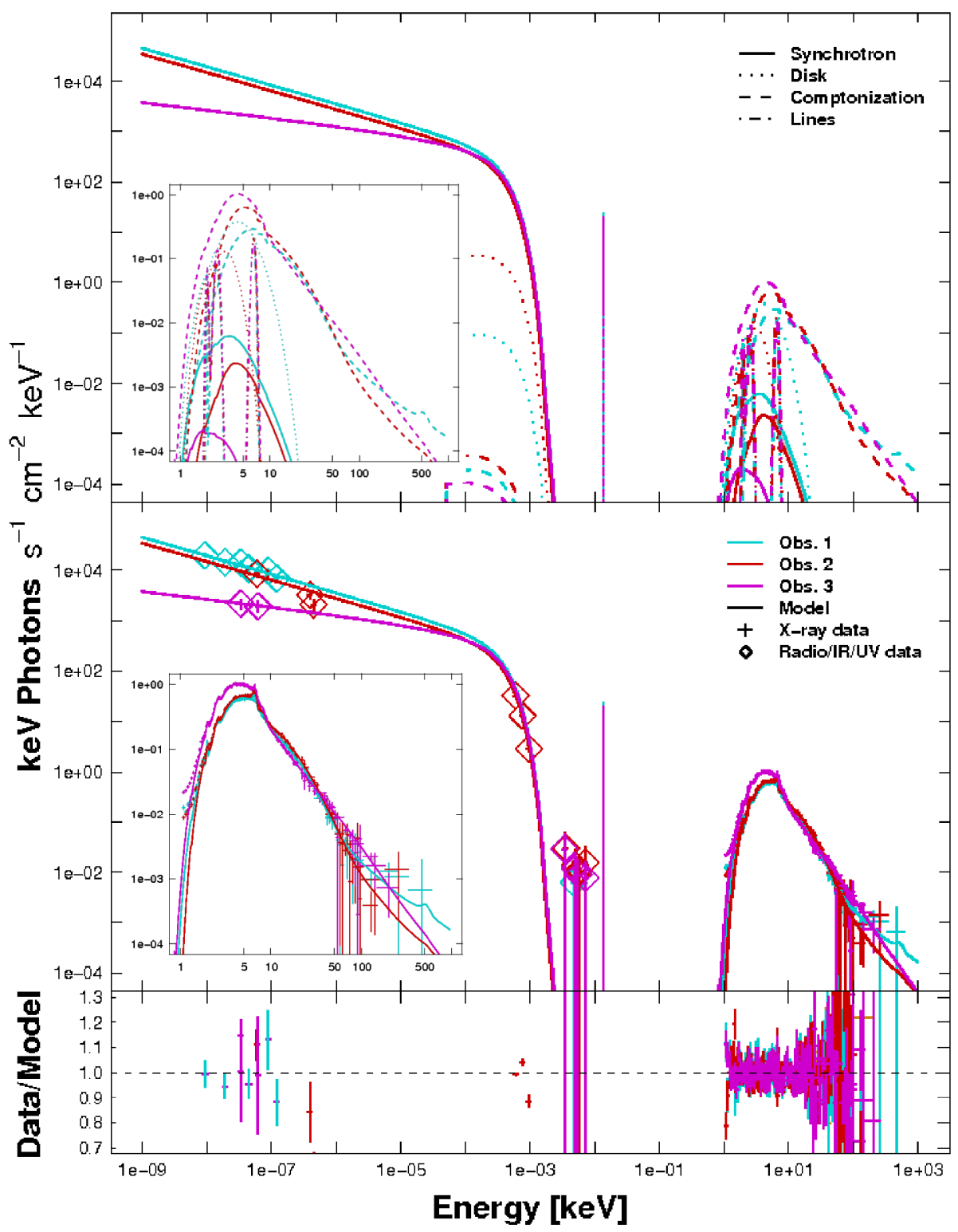

Figure 2: Synchrotron/Eqpair fits of the Cyg X-3 SEDs. The individual components are in the upper panel and the data and the full models in the lower panel of the ure. The X-ray spectra insets are included for greater clarity.

the Wolf-Rayet companion. However, a partial absorption component is strongly required to fit the data under $3 \mathrm{keV}$ and, as we have seen in e.g. Vilhu et al. (2003), a relatively simple absorption model is suitable for the fits in this work. The mean value of the absorption is $N_{H} \sim 3 \times 10^{22} \mathrm{~cm}^{-2}$ and the partially covered absorption $N_{H} \sim 9.5 \times 10^{22} \mathrm{~cm}^{-2}$ with a covering fraction $\sim 0.8$.

The absorption edge of highly ionized iron in $9.28 \mathrm{keV}$ (Verner et al. 1996) improves the fits substantially as is noted in Hjalmarsdotter et al. (2008) and it's therefore included in the model frozen at the default value. Cygnus X-3 harbors a large number of emission lines that are especially 
prominent in the high-resolution energy spectra, e.g. Paerels et al. (2000), but are also visible in the Swift/XRT data. Thus, we include three prominent lines in the model, these being the Fe K lines of iron $(\sim 6.5 \mathrm{keV}$ in the fits), $\mathrm{H}$-like sulphur $(\sim 2.5 \mathrm{keV}$ in the fits) and $\mathrm{H}$-like silicon $(\sim 2$ $\mathrm{keV}$ in the fits).

Different Comptonization models were tested to see which of them is most suitable for Cygnus $\mathrm{X}-3$. The reflion model gave the worst results, but since it picks the reflected emission lines and calculates the ionization parameter consistently it was used to estimate the ionization (4000 erg $\mathrm{cm} \mathrm{s}^{-1}$ for Obs 1. and Obs. 2 and $2000 \mathrm{erg} \mathrm{cm} \mathrm{s}^{-1}$ for Obs. 3). comptt has problems finding the electron temperature, $k T_{e}$, which is degenerate in the fits. Hence, we froze it to 30 $\mathrm{keV}$, since the hard X-ray spectra peak in that area. This model also gives much smaller values for the optical depth, $\tau$, than other models. compps gives better results $\left(\chi_{v}^{2} \sim 1.23\right)$ than comptt $\left(\chi_{v}^{2} \sim 1.45\right)$, but the optical depth pegs at the maximum allowed value in Obs. 1 and Obs. 2. Obs. 3 also has radically different parameter values compared to other observations. Including a reflection component to the compps model, as well as in the eqpair model, improves the fit. eqpair gives equally good results compared to compps $\left(\chi_{v}^{2} \sim 1.24\right)$ and in addition all the parameters behave more consistently. This model has been successfully implemented to Cygnus X-3 spectra, in Vilhu et al. (2003), Hjalmarsdotter et al. (2008) and Szostek \& Zdziarski (2008). The minimum and maximum Lorentz factors are frozen to their default values (1.3 and 1000 respectively) and the elemental abundances to the values given in Hamann \& Gräfener (2004). The soft photon compactness, $l_{s}$, is frozen to 10 following Gierliński et al. (1999). The fits give purely non-thermal spectra $\left(l_{n t} / l_{h}=1.00\right)$ and high values of optical depth, similar to compps. The hard-to-soft compactness, $l_{h} / l_{s}$, decreases throughout the observations, implying that the spectra soften with time. The disk temperature, $k T_{b b}$, varies between 0.7 and $1.3 \mathrm{keV}$, which is fairly typical for black hole binaries. We also fit eqpair with mono-energetic injection settings and found the fits to be equally good to those with power-law accelerated settings. The injected energy is $\sim 5.9 \mathrm{keV}$ in the observations and in this case the spectra are not fully non-thermal.

The evolution of the model component fluxes along these three data sets can be seen in Table 2. Right after the flare the synchrotron and disk fluxes dominate but as time goes on, inverse Comptonization of the disk photons in the base of the jet begins to dominate the total flux. Also, the synchrotron flux decreases with time as is expected as the major flare progresses.

We have successfully modeled the SEDs of Cygnus X-3 with a synchrotron model spanning from the radio to the soft X-rays and with Comptonization producing the higher energy emission. One of the questions that arises is what is the source of the relativistic particles that contributes to the upscattering of the photons. Due to the link with the radio, one of the possible mechanisms could be a magnetic reconnection event at the base of the jet which produces a steep distribution of relativistic electrons that are consequently sucked in the jet and accelerated by shocks to higher energies. This is still a work in progress and we continue to work and refine our analysis of these and other multiwavelength data sets for Cygnus X-3.

\section{Acknowledgements}

We thank Sergei Trushkin for providing the RATAN-600 data, Danny Steeghs for providing the PAIRITEL data, Guy Pooley for providing the Ryle data, Diana Hannikainen for valuable 
comments and finally Mike Nowak and John Houck for crucial help with ISIS. KIIK gratefully acknowledges SAO fellowship and Finnish Academy grant (project num. 125189) . MLM acknowledges support for this work from NASA under contract NAS8-03060 (CXC).

\section{References}

[1] Burrows, D. N., et al. 2005, Space Sci. Rev., 120, 165

[2] Coppi P. S., 1992, MNRAS, 258, 657

[3] Coppi P. S., 1999, ASPC, 161, 375

[4] Courvoisier T. J.-L., Beckmann V., Bourban G. et al. 2003, A\&A, 411, L343

[5] Dickey, J.M., \& Lockman, F.J. 1990, ARAA, 28, 215

[6] Giacconi R., Gorenstein P., Gursky H., Waters J. R., 1967, ApJ, 148, L119

[7] Gierliński, Zdziarski \& Poutanen, 1999, MNRAS, 309, 496

[8] Hjalmarsdotter L., Zdziarski A. A., Larsson S., Beckmann V., Mc- Collough M., Hannikainen D. C., Vilhu O., 2008, MNRAS, 384, 278

[9] Houck, J., C., 2002, ISIS: The Interactive Spectral Interpretation System. In Branduardi-Raymont, G., editor, High Resolution X-ray Spectroscopy with XMM-Newton and Chandra, Mul lard Space Science Laboratory of University Col lege, London.

[10] Houck \& Allen, 2006, ApJS, 167, 26H

[11] Lebrun F., Leray J., Lavocat P., Cretolle J., Arques M., et al., 2003, A\&A, 411, L141

[12] Lund N., et al. 2003, A\&A, 411, L231

[13] Martí J., Paredes J. M., Peracaula M., 2000, ApJ, 545, 939

[14] McCollough, M., et al. 1999, ApJ, 517, 951

[15] Paerels F., Cottam J., Sako M., Liedahl D. A., Brinkman A. C., van der Meer R. L. J., Kaastra J. S., Predehl P., 2000, ApJ, 533, L135

[16] Poutanen J., Svensson R., 1996, ApJ, 470, 249

[17] Predehl P., Burwitz V., Paerels F., Trümper J., 2000, A\&A, 357, L25

[18] Roming, P. W. A., et al. 2005, Space Science Reviews, 120, 95

[19] Rotschild et al., 1998

[20] Skinner G. \& Connell P., 2003, A\&A, 411, L123

[21] Szostek, A., Zdziarski, A., McCollough, M. 2008, MNRAS, 388, 100

[22] Szostek A., Zdziarski A. A., 2008, MNRAS, 386, 593

[23] Titarchuk, L., 1994, ApJ, 434, 31

[24] Tsuboi, M., et al. 2008, PASJ, 60, 465

[25] van Keerkwijk M. H., et al., 1992, Nature, 355, 703

[26] Vedrenne G., Roques J.P., Schonfelder V., et al, 2003, A\&A, 411, L63

[27] Verner, D. A., Ferland, G. J., Korista, K. T., Yakovlev, D. G., 1996, ApJ, 465, 487

[28] Vilhu O., et al., 2003, A\&A, 411, L405

[29] Zimmerman, E. R., Narayan, R., McClintock, J. E., Miller, J. M., 2005, ApJ, 618, 832 
Table 1: Selected parameters of the fits to data sets 1, 2 and 3.

\begin{tabular}{|c|c|c|c|c|c|}
\hline Component & Parameter & Unit & Obs. 1 & Obs. 2 & Obs. 3 \\
\hline Time Since Flare & & Days & 0.25 & 2.51 & 6.88 \\
\hline Ezdiskbb & $T_{\mathrm{bb}}$ & $\mathrm{keV}$ & $1.75_{-.12}^{+.11}$ & $1.51_{-.25}^{+.22}$ & $1.51_{-.10}^{+.05}$ \\
\hline \multirow[t]{2}{*}{ ComptT } & $T_{0}$ & $\mathrm{keV}$ & $2.95_{-.38}^{+.41}$ & $2.36_{-.39}^{+.40}$ & $2.43_{-1.37}^{+.47}$ \\
\hline & $\tau$ & & $0.38_{-.04}^{+.04}$ & $0.30_{-.03}^{+.03}$ & $0.70_{-.04}^{+.03}$ \\
\hline \multirow[t]{3}{*}{ Sync } & $\Gamma$ & & $1.75_{-.10}^{+.11}$ & $1.73_{-.03}^{+.03}$ & $1.50_{-.06}^{+.04}$ \\
\hline & $E_{\text {cut }}$ & $\mathrm{TeV}$ & $31_{-9}^{+12}$ & $40_{-10}^{+6}$ & $42_{-15}^{+10}$ \\
\hline & $N_{s}^{a}$ & $10^{5} \mathrm{~cm}^{-2} \mathrm{GeV}^{-1}$ & $42_{-14}^{+20}$ & $30_{-6}^{+6}$ & $2.3_{-1.3}^{+2.1}$ \\
\hline Confidence & $\chi_{v}^{2} /$ d.o.f. & & $1.35 / 193$ & $1.77 / 174$ & $1.24 / 190$ \\
\hline \multirow[t]{5}{*}{ CompPS } & $k T_{e}$ & $\mathrm{keV}$ & $15.85_{-.07}^{+.07}$ & $16.96_{-.08}^{+.08}$ & $\overline{107_{-15}^{+11}}$ \\
\hline & $\gamma_{\min }$ & & $1.40_{-.01}^{+.01}$ & $1.48_{-.02}^{+.02}$ & $3.18_{-.11}^{+.59}$ \\
\hline & $k T_{b b}$ & $\mathrm{keV}$ & $1.07_{-.02}^{+.01}$ & $1.07_{-.03}^{+.01}$ & $1.49_{-.04}^{+.04}$ \\
\hline & $\tau$ & & $3.00_{-.46}^{+.00}$ & $3.00_{-.02}^{+.00}$ & $0.18_{-.03}^{+.04}$ \\
\hline & $\Omega / 2 \pi$ & & $0.48_{-.01}^{+.02}$ & $0.49_{-.14}^{+.02}$ & $0.00_{-.00}^{+.28}$ \\
\hline \multirow[t]{3}{*}{ Sync } & $\Gamma$ & & $1.66_{-.01}^{+.17}$ & $1.72_{-.04}^{+.01}$ & $1.35_{-.35}^{+1.03}$ \\
\hline & $E_{\text {cut }}$ & $\mathrm{TeV}$ & $1_{-0}^{+29}$ & $26_{-10}^{+2}$ & $12_{-11}^{+88}$ \\
\hline & $N_{s}^{a}$ & $10^{5} \mathrm{~cm}^{-2} \mathrm{GeV}^{-1}$ & $32_{-4}^{+22}$ & $28_{-1}^{+1}$ & $1.5_{-1.3}^{+3.1}$ \\
\hline Confidence & $\chi_{v}^{2} /$ d.o.f. & & $1.08 / 194$ & $1.63 / 175$ & $0.99 / 191$ \\
\hline \multirow[t]{6}{*}{ Eqpair } & $k T_{b b}$ & $\mathrm{eV}$ & $1318_{-2}^{+.2}$ & $852_{-96}^{+42}$ & $758_{-1}^{+1}$ \\
\hline & $\mathrm{l}_{\mathrm{nt}} / \mathrm{l}_{\mathrm{h}}$ & & $1.00_{-.38}^{+.00}$ & $1.00_{-.71}^{+.00}$ & $1.00_{-.17}^{+.00}$ \\
\hline & $\mathrm{l}_{\mathrm{h}} / \mathrm{l}_{\mathrm{s}}$ & & $0.88_{-.01}^{+.02}$ & $0.66_{-.01}^{+.01}$ & $0.34_{-.01}^{+.01}$ \\
\hline & $\tau$ & & $3.83_{-.15}^{+.15}$ & $3.93_{-.10}^{+.01}$ & $1.33_{-.05}^{+.05}$ \\
\hline & $\Gamma$ & & $2.36_{-.03}^{+.03}$ & $4.17_{-.16}^{+.16}$ & $3.69_{-.06}^{+.07}$ \\
\hline & $\Omega / 2 \pi$ & & $0.62_{-.16}^{+.04}$ & $0.00_{-.00}^{+.02}$ & $0.81_{-.03}^{+.02}$ \\
\hline \multirow[t]{3}{*}{ Sync } & $\Gamma$ & & $1.73_{-.10}^{+.11}$ & $1.74_{-.03}^{+.02}$ & $1.30_{-.05}^{+.08}$ \\
\hline & $E_{\text {cut }}$ & $\mathrm{TeV}$ & $32_{-10}^{+13}$ & $46_{-10}^{+13}$ & $13_{-12}^{+6}$ \\
\hline & $N_{s}^{a}$ & $10^{5} \mathrm{~cm}^{-2} \mathrm{GeV}^{-1}$ & $39_{-13}^{+18}$ & $31_{-6}^{+5}$ & $1.2_{-.3}^{+.3}$ \\
\hline Confidence & $\chi_{v}^{2} /$ d.o.f. & & $1.12 / 193$ & $1.60 / 174$ & $1.01 / 190$ \\
\hline
\end{tabular}

a $N_{s}=\frac{A_{e} V_{S}}{4 \pi d^{2}}$. $A_{e}$ is the normalization of the nonthermal electron momentum distribution, $d$ is the distance to the source and $V_{S}$ is the synchrotron emitting volume. 
Table 2: Bolometric fluxes of the different wavelength areas and different components of the eqpair fits.

\begin{tabular}{llllll} 
Component & Parameter & Unit & Obs. 1 & Obs. 2 & Obs. 3 \\
\hline Absorbed Flux & $F_{\mathrm{X}, \mathrm{bol}}^{a}$ & $10^{-9} \mathrm{erg} \mathrm{cm}^{-2} \mathrm{~s}^{-1}$ & 9.41 & 9.99 & 12.3 \\
& $F_{\mathrm{I} \text { bol }}^{c}$ & $10^{-9} \mathrm{erg} \mathrm{cm}^{-2} \mathrm{~s}^{-1}$ & 0.01 & 0.01 & 0.01 \\
& $F_{\mathrm{R}, \mathrm{bol}}$ & $10^{-9} \mathrm{erg} \mathrm{cm}^{-2} \mathrm{~s}^{-1}$ & 0.32 & 0.25 & 0.24 \\
& $F_{\text {Sync }} / F_{X+R+I}$ & & 0.04 & 0.03 & 0.02 \\
& $F_{\text {Disk }} / F_{X+R+I}$ & & 0.29 & 0.04 & 0.00 \\
& $F_{\text {Comp }} / F_{X+R+I}$ & & 0.65 & 0.90 & 0.95 \\
& $F_{\text {Line }} / F_{X+R+I}$ & & 0.02 & 0.03 & 0.03 \\
\hline Unabsorbed Flux & $F_{\mathrm{X}, \text { bol }}^{a}$ & $10^{-9} \mathrm{erg} \mathrm{cm}^{-2} \mathrm{~s}^{-1}$ & 18.0 & 21.0 & 24.5 \\
& $F_{\mathrm{I}, \text { bol }}^{b}$ & $10^{-9} \mathrm{erg} \mathrm{cm}^{-2} \mathrm{~s}^{-1}$ & 7.76 & 7.93 & 4.30 \\
& $F_{\mathrm{R}, \text { bol }}^{c}$ & $10^{-9} \mathrm{erg} \mathrm{cm}^{-2} \mathrm{~s}^{-1}$ & 1.26 & 1.02 & 1.12 \\
& $F_{\text {Sync }} / F_{X+R+I}$ & & 0.27 & 0.25 & 0.15 \\
& $F_{\text {Disk }} / F_{X+R+I}$ & & 0.40 & 0.16 & 0.00 \\
& $F_{\text {Comp }} / F_{X+R+I}$ & & 0.31 & 0.56 & 0.82 \\
& $F_{\text {Line }} / F_{X+R+I}$ & & 0.02 & 0.03 & 0.03 \\
\hline
\end{tabular}

\footnotetext{
a X-ray bolometric flux 1-1000 keV.

b IR/Optical/UV bolometric flux $12-3100 \AA$.

c Radio bolometric flux $1 \mathrm{GHz}-1 \mathrm{PHz}$.
} 